\title{
The Magnet System of Wendelstein 7-X Stellarator in Operation
}

\author{
Konrad Risse a , Thomas Rummel ${ }^{\text {a }}$, Hans-Stephan Bosch ${ }^{\text {a }}$, Victor Bykov ${ }^{\mathrm{a}}$, André Carls ${ }^{\mathrm{a}}$, Frank \\ Füllenbach ${ }^{\text {a }}$, Thomas Mönnich ${ }^{\text {a }}$, Michael Nagel ${ }^{\text {a }}$, Matthias Schneider ${ }^{\text {a }}$, W7-X Team ${ }^{\mathrm{a}}$ \\ ${ }^{a}$ Max Planck Institute for Plasma Physics, Wendelsteinstr. 1, 17491 Greifswald, Germany
}

\begin{abstract}
The Wendelstein 7-X stellarator (W7-X), one of the largest stellarator fusion experiments, will start the second plasma operation phase in August 2017 at the Max Planck Institute for Plasma Physics in Greifswald, Germany. The main objective of the experiment is to prove the reactor relevance of the stellarator design. Recently the inner wall shielding of the plasma vessel was completed in a 12 month break and a first, inertially cooled divertor has been installed. The coming plasma experiments can be executed with increased ECRH heating power up to $9 \mathrm{MW}$ and a plasma pulse length up to $100 \mathrm{~s}$ limited by a total energy input of $80 \mathrm{MJ}$.

The W7-X experiment has a superconducting magnet system with 50 non-planar and 20 planar coils grouped in five equal modules and electrically connected in seven circuits with 10 coils each. Seven power supplies provide individual coil currents in the seven circuits. Two normal conductive copper coil systems can be operated in parallel to the superconducting coils. The paper reports about the experiences with the superconducting magnet system during the first plasma operation phase, about the commissioning for the coming second phase and results from mechanical sensor evaluation.
\end{abstract}

Keywords: fusion, stellarator, superconducting magnets, magnetic field, magnets

\section{Introduction}

After a 12 month break the Wendelstein 7-X stellarator (W7-X), will start the second plasma operation phase in August 2017. The main objective of the fusion experiment is to prove the reactor relevance of the stellarator design. Especially the inner wall shielding of the plasma vessel was completed in a 12 month break. The coming plasma experiments can be executed with increased Electron Cyclotron Resonance Heating (ECRH) power up to $9 \mathrm{MW}$ and a plasma pulse length up to $100 \mathrm{~s}$ limited by a total energy input of $80 \mathrm{MJ}$.

The W7-X experiment has a superconducting magnet system which provides the main magnetic field for the particle confinement. The normally conducting copper coil systems of the trim coils and the control coils can be operated in parallel to the superconducting coils.

The superconducting magnet system was thoroughly tested during a commissioning period in July 2017 to be ready for plasma operation in August 2017. The normally conducting trim coils and control coils are now commissioned for full current operation.

\section{W7-X magnet system components}

\subsection{Superconducting magnet system}

The superconducting magnet system of W7-X consists of 50 non-planar and 20 planar coils grouped in five equal modules, electrically connected in seven circuits with 10 coils each. The main magnetic stellarator field is provided by five different Non-Planar Coil (NPC) types and can be modified by two different Planar Coil (PC) types, see Fig. 1 [1].

Seven power supplies provide individual coil currents in the seven circuits. A quench detection system checks permanently the superconducting system with regard to the occurrence of a quench. In case of a quench the magnet safety system will be activated and a set of switches leads the current into discharge resistors.

Specially developed high temperature superconducting Current Leads (CL) feed the current into the cryostat by bridging the temperature difference from room temperature down to the $4 \mathrm{~K}$ level.

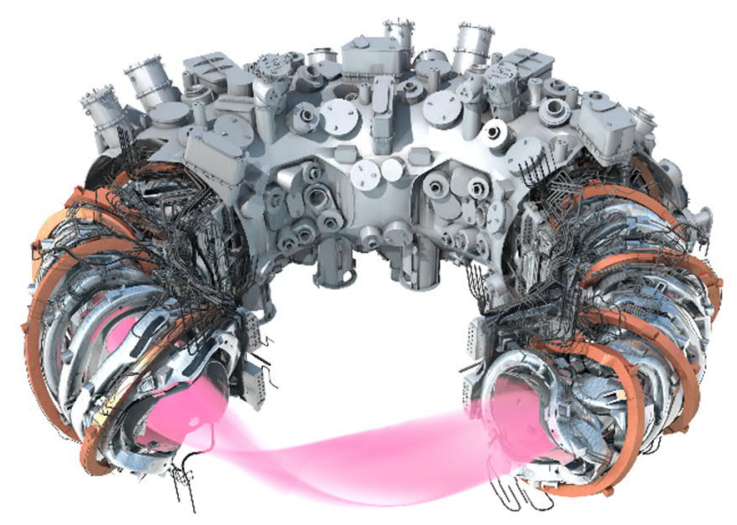

Fig. 1. Schematic view on W7-X cryostat with superconducting magnets.

The superconducting coils and their interconnecting bus bar system use the same Cable in Conduit Conductor (CICC). The conductor comprises a cable with $243 \mathrm{NbTi}$ strands enveloped by an Aluminum (Al) jacket. The NPC are made of 108 turns in 6 double layers and the PC have 36 turns in 3 double layers. The vacuum pressure impregnated winding packs are embedded into a heavy stainless steel cast casing. The coils are screwed onto a central ring support structure and additionally interconnected by support elements in between the single coils. The coils and the support structure are equipped 
with an extensive set of sensors e.g. temperature measurements on the He-inlets and outlets on the winding pack as well as on the casing, strain gauges on loaded positions on the support structure and distance sensors to measure distance changes between coils.

\subsection{Trim coil system}

Five normally conductive copper coils allow a fine tuning of the main magnetic field during plasma operation. The coils are mounted on the outer cryostat wall, one coil per each of the five W7-X modules, see Fig. 2. Due to construction space restrictions two different coil types were developed: four Type A coils and one coil of Type B. The coils are designed to operate with a nominal current of $1.8 \mathrm{kA}$ for Type A and $1.95 \mathrm{~A} \mathrm{kA}$ for Type B. The electrical insulation of the coils is designed to withstand the nominal voltages of $200 \mathrm{~V}$ and the related test voltages of $2 \mathrm{kV}$ DC to ground while maintaining an insulation resistance of $1 \mathrm{G} \Omega$ [2].

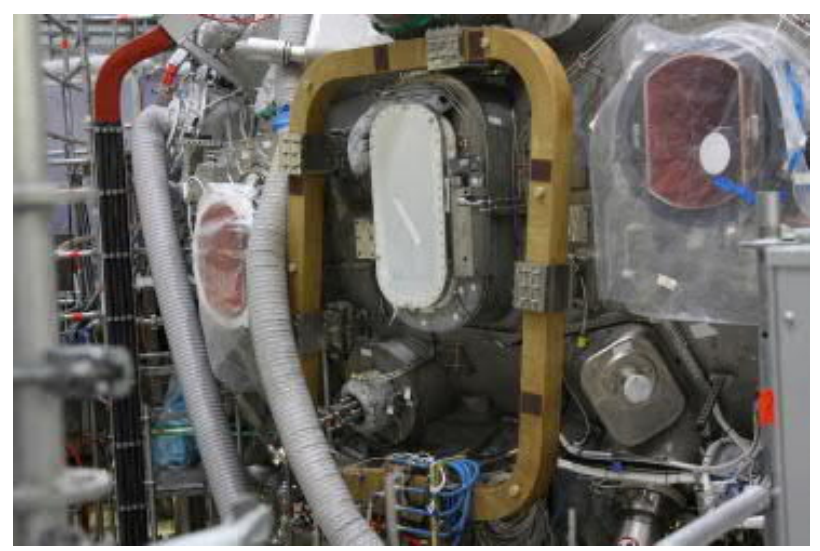

Fig. 2. Trim coil type B mounted on cryostat.

\subsection{Control coil system}

The control coil system with 10 coils, positioned in the plasma vessel behind the divertor units, allows the wider distribution of heat loads on the divertor and the modification of symmetry breaking error fields. For this purpose each coil can be individually loaded with a DC current from - $2500 \mathrm{~A}$ to $2500 \mathrm{~A}$ and additionally with a superimposed AC component with $625 \mathrm{~A}$ between $1 \mathrm{~Hz}$ to $20 \mathrm{~Hz}$ frequency [3].

\section{Operation of the superconducting magnets}

\subsection{Experiences from first plasma operation phase}

After the commissioning of the superconducting magnet system in summer 2015, the first plasma operation phase (OP) started in December 2015 and ended in March 2016 [4]. The first operation phase of W7-X was performed with a magnetic field of $2.5 \mathrm{~T}$ at the area where the ECRH waves should be absorbed by the plasma. To provide the magnetic field, the magnet system was operated with the magnetic field configuration "OP1.1 Limiter" which was defined with $12.8 \mathrm{kA}$ in the five NPC circuits and $5 \mathrm{kA}$ in the two $\mathrm{PC}$ circuits respectively. The superconducting magnets were energized on 31 operation days for about 180 hours. The OP1.1 period ended on $10^{\text {th }}$ March 2016 after more then 900 plasma experiments, finally heated up with ECRH energies up to $4 \mathrm{MJ}$ with electron temperatures of $8 \mathrm{keV}$ and discharge durations of $6 \mathrm{~s}$. The magnets were operated with a temperature margin of $1 \mathrm{~K}$ e.g. $5.16 \mathrm{~K}$ was the maximum allowed temperature criterion for the NPC operation in OP1.1. No quench happened on the magnets so far. The thermal behaviour of the magnet system during ramping up and during the plateau phases was excellent. $0.2 \mathrm{~K}$ temperature increase was measured during ramping up with $30 \mathrm{~A} / \mathrm{s}$. The temperature increase during plateau phase was about $38 \mathrm{mK}$ on one NPC due to the ohmic heating which corresponds to $2.9 \mathrm{nOhm}$ resistance resulting from the low resistance superconductor joints in the coil $[5,6]$.

The initial magnetic flux surface measurements confirm so far the existence of good and nested magnetic flux surfaces up to the nominal field strength of $2.5 \mathrm{~T}$ without significant errors. The measured position and shape of the magnetic flux surfaces are in good agreement with simulations and validate the magnetic field models [7].

\subsection{Electrical insulation monitoring}

High voltage (HV) tests carried out on the magnet system shall prove the capability of the electrical insulation system to handle the voltage during a fast discharge of the magnet system. HV tests are performed regularly before and after the plasma operation phases at warm and cold conditions. The measured leak current will be reported after $60 \mathrm{~s} \mathrm{HV}$ plateau phase together with the passed / not passed judgment.

A test program started in spring 2017 to measure the insulation resistance under defined conditions. The test voltage of $500 \mathrm{~V}$ is applied for $120 \mathrm{~s}$, the resulting current is recorded by an electrometer which also provides the voltage. The current is limited by a series resistor of $10 \mathrm{M} \Omega$. Important is that the magnet system is shortened over a longer period, to allow the complete discharging of the insulation system.

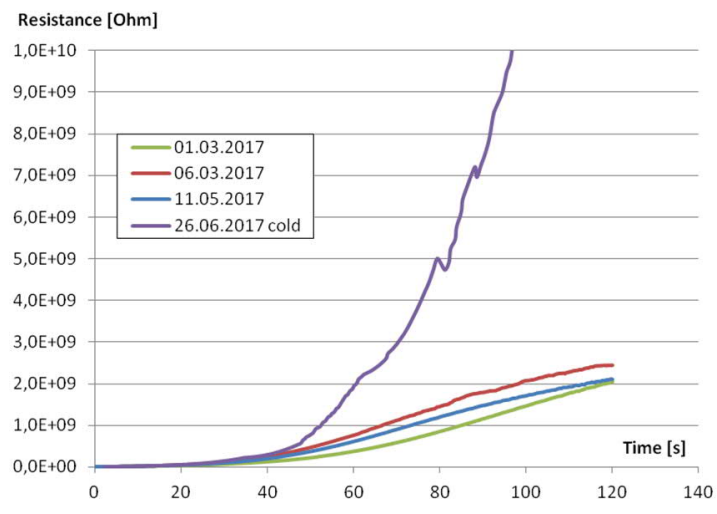

Fig. 3. Insulation resistance on NPC 1 type measured with $500 \mathrm{~V}$ at different days at warm and cold conditions.

The measured current in the first minutes of a HV test is a summary of the leak current representing the insulation resistance, the absorption current and the capacitative load current. Fig. 3 shows the measured values on the NPC1 magnet system on different days at warm conditions and one set with cold components (at 
$10 \mathrm{~K})$. The resulting curves at warm conditions are similar to each other with an insulation resistance of about $2 \mathrm{G} \Omega$ while the curve at cold cryostat conditions differs a lot and shows much higher resistance values. The signal over $4 \mathrm{G} \Omega$ (less than $125 \mathrm{nA}$ ) shows larger influences from noise signals. A long term observation of the electrical load behaviour according Fig. 3 should allow an assessment if the insulation system will start to develop a degradation.

\subsection{Optimization of discharge resistors}

The discharge resistors, as part of the magnet safety system, determine the levels and the time behaviour of voltage and current during the discharge process. The existing nickel discharge resistors of the NPC's were rearranged to reduce the resistance from $148 \mathrm{~m} \Omega$ to $74 \mathrm{~m} \Omega$ which results in a longer discharge period with a discharge voltage decreased by about one third. The new discharge resistor configuration for the NPC considers the required magnet field configurations with $2.5 \mathrm{~T}$ on the magnetic axis, the resulting hot spot temperature in the superconductor in case of a quench and the maximum allowed temperature of the resistors.

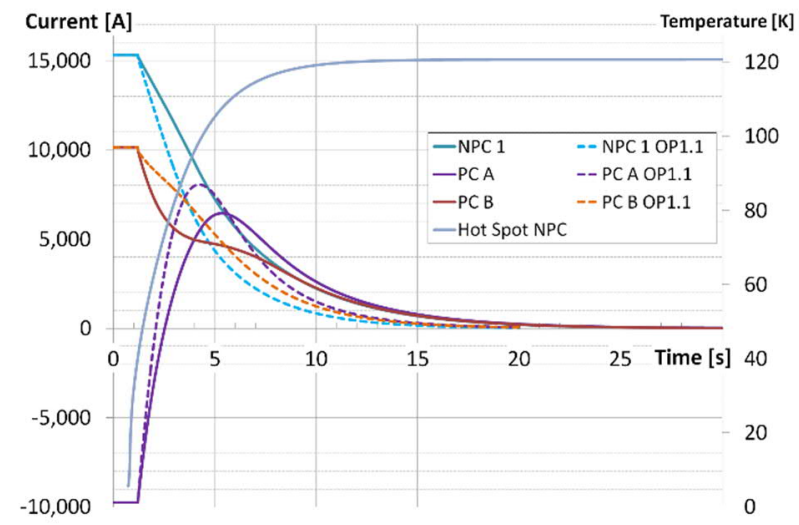

Fig. 4. NPC 1, PC A and PC B currents during fast discharge of $2.5 \mathrm{~T}$ Low shear configuration compared with former resistor configuration, added Hot spot calculation for NPC 1.

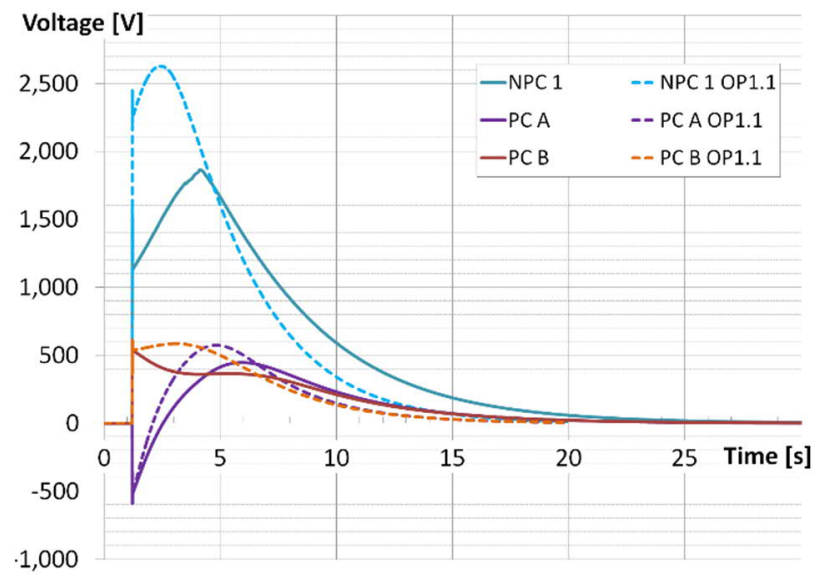

Fig. 5. NPC 1, PC A and PC B voltages during fast discharge of $2.5 \mathrm{~T}$ Low shear configuration compared with former resistor configuration.

The discharge process is analyzed for all 10 magnetic field reference configurations within an ANSYS Simplorer model. The Low Shear case is the field configuration which contains with $440 \mathrm{MJ}$ the highest energy during a $2.5 \mathrm{~T}$ operations. Fig. 4 presents the results from the ANSYS Simplorer model with currents for NPC 1, PC A and B during a fast discharge. The currents for the NPC 2 - 5 types are not shown in Fig.4 because of similar current curves. The PC A carries minus $9.76 \mathrm{kA}$ while the PC B carry $10.16 \mathrm{kA}$. The dashed lines represent the current curves with the former resistor configuration. It becomes visible that the discharge process for the NPC works with reduced speed in comparison to the former status. The PC A show a fast ramp down with zero crossing up to a positive current of $6.4 \mathrm{kA}$ due to the inductive coupling to the NPC. The decreased $\mathrm{di} / \mathrm{dt}$ rate results in a reduced discharge voltage on the NPC, see Fig. 5. The voltage on the NPC 1 system (representing the max. voltage on all NPC) drops down from $2.8 \mathrm{kV}$ to $1.8 \mathrm{kV}$.

A fast discharge is triggered in case of a quench in the superconducting magnets. The current is decreasing according to the curves described in Fig.4. The quenched superconductor section becomes normally conducting and develops a heating zone where the temperature increases - a so called hot spot area. Fig.4 contains additionally the results from a hot spot calculation inside the W7-X CICC considering isochors and isobar conditions in the conductor by neglecting the heat conducting into the surrounding electrical insulation. The hot spot temperature went up to $120 \mathrm{~K}$ which is acceptable for NbTi based superconductors. The calculations considers at the beginning a quench detection time of $150 \mathrm{~ms}$ and a time of $350 \mathrm{~ms}$ to switch the current into the circuit with the discharge resistors.

\section{Preparation for OP1.2a plasma phase}

\subsection{Commissioning of superconducting magnets}

The commissioning for OP1.2a took place in July 2017 with the purpose to test several changes in the control software and to test the changes made on the discharge resistors (see chapter 3.3). One main change in the control system was the increase of the current ramp up / down speed from $30 \mathrm{~A} / \mathrm{s}$ up to $40 \mathrm{~A} / \mathrm{s}$. A faster controlled ramp down of the magnet system optimizes the system behaviour e.g. in case of a Helium leakage in the cryostat. Additionally a new data acquisition system was commissioned during these days.

The well known magnetic field configuration "OP1.1 Limiter" with $12.8 \mathrm{kA}$ in the five NPC circuits and respectively $5 \mathrm{kA}$ in the two $\mathrm{PC}$ circuits was used for the commissioning tests. The commissioning has been started with several 500 A pulses to check the balancing of the Quench Detection System (QDS) and to check if there is an influence due to the increased ramp speed. Following tests were performed at $2 \mathrm{kA}, 6 \mathrm{kA}, 10 \mathrm{kA}$ and $12.8 \mathrm{kA}$ levels in the NPC while the current in the PC was adjusted accordingly:

- Different ramp up and ramp down scenarios finally with $40 \mathrm{~A} / \mathrm{s}$.

- The magnet safety system functionality was tested with fast discharges up to the $10 \mathrm{kA}$ level. 
- The thermal behaviour of the superconducting components and the data from the mechanical sensors (strain gauges, distance sensors and contact sensors) were online monitored during the commissioning.

Finally following results were received from the commissioning of the superconducting magnets:

- The QDS is not significantly influenced by the increased ramp speed of $40 \mathrm{~A} / \mathrm{s}$, a new balancing of the QD units was not necessary.

- The new data acquisition system worked well, minor issues will be solved soon.

- The changes in the control software of the power supplies worked well, the maximum ramp rate of $40 \mathrm{~A} / \mathrm{s}$ could not be reached completely clarification is ongoing.

- The curves for current and voltage during the fast discharge were checked and compared with the results from the ANSYS Simplorer model.

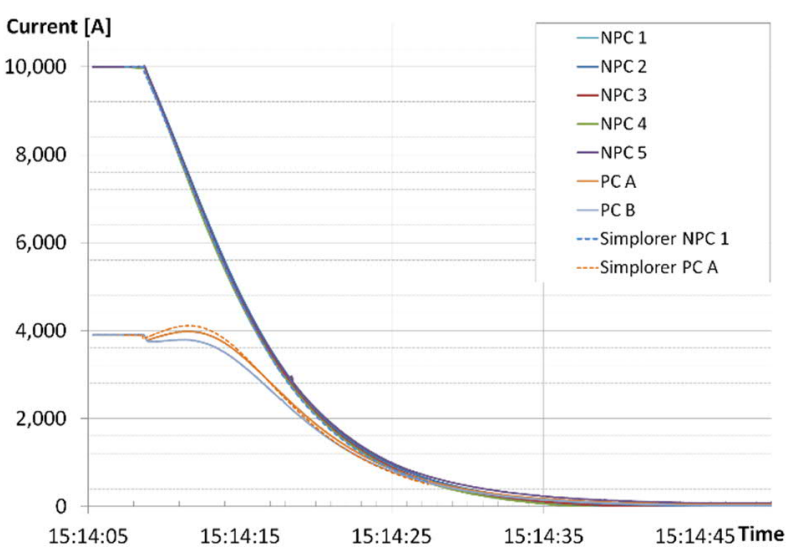

Fig. 6. Measured current data during a fast discharge in comparison to the ANSYS Simplorer data for NPC 1 and PC A.

Fig. 6 presents the current curves for the five NPC and the two PC circuits during a fast discharge on the $6^{\text {th }}$ July
2017 from the $10 \mathrm{kA}$ plateau of the "OP1.1 Limiter" field configuration. The current curves modeled with ANSYS Simplorer are also shown in Fig. 6 with dotted lines (other coil types not shown because of clarity). While the dotted line for PC A is visible in the near vicinity of the measured data line of PC A, the dotted line for NPC 1 type is hidden behind the measured current lines. This is the proof that the newly arranged discharge resistors works well and the accuracy of the ANSYS Simplorer model is in good accordance with the measured values.

A similar test program as described in this chapter is planned for the new magnetic field configurations described in Table 2, but the tests will be performed directly before the physics program will use this configuration.

\subsection{Mechanical integrity}

The magnet system commissioning was used to update the global finite element model for the magnet system, to review the results of the previous operation phase, to predict and verify the repetitive behaviour of the magnet system with its non-linear supports [8].

The magnet system components that require special attention e.g. highly pre-stressed bolts, planar coils, etc were defined and closely monitored during the current ramps. This process is based on the signals from the extended mechanical instrumentation system introduced in W7-X [9]. The measurement data are processed by a specially developed online monitoring MATLAB tool [10]. The tool performs a fast reliable assessment of the structural response during commissioning and operation in parallel with other relevant data and parameters of the W7-X machine. Both the magnet system cool down process and the current operation regime have been continuously supervised. The configuration of electronics has been changed during the commissioning process to deliver required signal sets.

A comparison between measurements for repetitive cycles before and after 1 year assembly phase, shows relatively good agreements (see Table 1) with a few exceptions which are under detailed analysis now.

Table 1. Fragment of typical table to compare measurement results with FE prediction (bold) in percentage and absolute value, and to confirm repetitive mechanical behavior of magnet system (measurement result in italic): mutual coil displacement measurements for one half module in $\mathrm{mm}$.

\begin{tabular}{|c|c|c|c|c|c|c|c|c|c|c|c|c|c|}
\hline \multirow[t]{2}{*}{ Sensor name } & \multirow{2}{*}{$\begin{array}{l}\mathrm{FE} \\
\text { results } \\
{[\mathrm{mm}]}\end{array}$} & \multicolumn{2}{|c|}{$\begin{array}{l}\text { 06-Jul-2015 } \\
11: 13-12: 05\end{array}$} & \multicolumn{3}{|c|}{$\begin{array}{l}\text { 13-Jan-2016 } \\
\text { 12:45-18:37 } \\
{[\mathrm{mm}}\end{array}$} & \multicolumn{3}{|c|}{$\begin{array}{l}\text { 10-Mar-2016 } \\
12: 10-17: 55\end{array}$} & \multicolumn{4}{|c|}{$\begin{array}{l}\text { 07-Jul-2017 } \\
11: 46-12: 00\end{array}$} \\
\hline & & {$[\mathrm{mm}]$} & Diff & $\%$ & ] & Diff & $\%$ & {$[\mathrm{~mm}]$} & Diff & $\%$ & {$[\mathrm{~mm}]$} & Diff & $\%$ \\
\hline AAT11CG020 & 2.1 & 1.7 & -0.43 & -20 & 1.4 & -0.71 & -33 & 1.3 & -0.81 & -38 & 1.6 & -0.55 & -26 \\
\hline AAT11CG021 & -1.5 & -2.4 & -0.86 & 56 & -2.2 & -0.61 & 40 & -2.5 & -0.97 & 63 & -2.2 & -0.61 & 39 \\
\hline AAT11CG022 & 8.0 & 8.3 & 0.3 & 4 & 8.0 & 0.01 & 0 & 8.0 & -0.07 & -1 & 8.3 & 0.34 & 4 \\
\hline AAT11CG023 & -1.8 & -1.4 & 0.37 & -21 & -1.4 & 0.4 & -22 & -1.2 & 0.56 & -32 & -1.5 & 0.3 & -17 \\
\hline $\begin{array}{l}\text { AAT11CG025 } \\
\text { AAT11CG025 }\end{array}$ & -9.6 & -11.9 & -2.24 & 23 & -11.6 & -1.95 & 20 & -11.7 & -2.02 & 21 & -11.8 & -2.2 & 23 \\
\hline r & -3.8 & -3.5 & 0.28 & -7 & -3.4 & 0.44 & -12 & -3.4 & 0.43 & -11 & -3.6 & 0.22 & -6 \\
\hline AAT11CG026 & 0.27 & -0.42 & -0.7 & - & -0.54 & -0.82 & - & -0.55 & -0.83 & - & -0.48 & -0.76 & - \\
\hline
\end{tabular}


Several accompanying measurements have been prepared and successfully performed to check the structural behaviour of the normally conducting trim coils with full operational currents, with and without energizing of superconducting coils.

An extended set of $2.5 \mathrm{~T}$ magnetic configurations with many new regimes requested by physicists is expected to be successfully carried out in OP1.2. The new operation phase gives an additional possibility to benchmark the numerical model and to be prepared for the next, more demanding phases.

\subsection{Magnetic field configurations forOP1.2a}

The next plasma operation phase OP1.2a will start in August 2017 with experiments executed with increased ECRH heating power up to $9 \mathrm{MW}$ and a plasma pulse length up to $100 \mathrm{~s}$ limited by a total energy input of $80 \mathrm{MJ}$. The physics program for OP1.2a requires 8 different magnetic field configurations with $2.5 \mathrm{~T}$ on the magnetic axis. Table 2 lists the magnetic field configurations which are specially challenging regarding mechanical loads and the discharge process because of higher currents and currents with minus directions [11].

Table 2. Challenging magnetic field configurations in OP1.2a with currents in the seven magnet circuits.

\begin{tabular}{lllll}
\hline System & $\begin{array}{l}\text { High } \\
\text { iota } \\
{[\mathrm{kA}]}\end{array}$ & $\begin{array}{l}\text { Low } \\
\text { iota } \\
{[\mathrm{kA}]}\end{array}$ & $\begin{array}{l}\text { Inward } \\
\text { shift } \\
{[\mathrm{kA}]}\end{array}$ & $\begin{array}{l}\text { Field } \\
\text { reversal } \\
{[\mathrm{kA}]}\end{array}$ \\
\hline NPC 1 & 14.9 & 12.2 & 13.1 & -13.5 \\
NPC 2 & 14.9 & 12.2 & 12.9 & -13.5 \\
NPC 3 & 14.9 & 12.2 & 13.2 & -13.5 \\
NPC 4 & 14.9 & 12.2 & 14.6 & -13.5 \\
NPC 5 & 14.9 & 12.2 & 14.7 & -13.5 \\
PS A & -10.3 & 9.2 & 4.1 & 0 \\
PS B & -10.3 & 9.2 & -8.17 & 0 \\
\hline
\end{tabular}

The discharge process is modelled in detail for all the required magnetic field configuration, the resulting discharge voltage on the NPC goes up to $1.7 \mathrm{kV}$ for the High iota configuration.

\section{Summary}

The superconducting magnet system was operated during the first plasma operation phase for about 180 hours on 31 operation days. No quench occurred up to now; the thermal safety margin of $1 \mathrm{~K}$ was kept all time. After a 12 month break, with several changes on the control software and reconfigured discharge resistors, the superconducting magnet system of W7-X was successfully commissioned at the beginning of July 2017. Special attention was paid to the fast discharge process of the magnets. Analysis had shown that the current and voltage curves are in good correlation with the electrical model in ANSYS Simplorer. The commissioning tests also validated changes made on the control systems of the power supplies. Mechanical sensor analysis was performed by an online monitoring with a specially developed MATLAB tool in parallel to the commissioning. A comparison between measurements for repetitive cycles shows relatively good agreements. The normally conducting trim coils and control coils are also available with full current performance for the next plasma operation phase OP1.2a which starts end of August 2017.

\section{Acknowledgments}

Author would like to thank colleagues from the W7-X operations division, the magnet system group members and the complete W7-X team for their support.

This work has been carried out within the framework of the EUROfusion Consortium and has received funding from the Euratom research and training program 20142018 under grant agreement No 633053. The views and opinions expressed herein do not necessarily reflect those of the European Commission.

\section{References}

[1] T. Rummel, The superconducting magnet system of the stellarator Wendelstein 7-X, IEEE Trans. on Plasma Science 40 (3) (2012) 769-776.

[2] F. Füllenbach et al., The trim coils for the Wendelstein 7-X magnet system, presented at SOFT 2016, to be published in Fusion Eng. Design.

[3] F. Füllenbach et al., Final test of the w7-X control coils power supply and its integration into the overall control environment, Fusion Engineering and Design 82 (5-14) (2007) 1391-1395.

[4] H. S. Bosch et al., Final integration, commissioning and start of the Wendelstein 7-X stellarator operation, Nuclear Fusion 57 (2017) 116015.

[5] M. Nagel et al., Cryogenic commissioning, cool down and first magnet operation of Wendelstein 7-X, IOP Conf. Series: Materials Science and Engineering 171 (2017) 012050.

[6] K. Risse et al., Wendelstein 7-X Commissioning of the Superconducting Magnet System, IEE Transactions on Applied Superconductivity 26 (4) (2016) 4202004.

[7] M. Otte et al., Setup and initial results from the magnetic flux surface diagnostics at Wendelstein 7-X, Plasma Phys. Control. Fusion 58, (2016) 064003.

[8] V. Bykov et al., Mechanical Monitoring Issues in Preparation to Next Step of W7-X Operation, presented at SOFE 2017, to be published in Transaction on Plasma Science.

[9] V. Bykov et al., Wendelstein 7-X mechanical instrumentation system for commissioning and operation, Fusion Science and Technology, 68 (2015) 267-271.

[10] A. Carls et al., A structural integrity monitoring tool for Wendelstein 7-X, presented at SOFT 2016, to be published in Fusion Eng. Design.

[11] T. Rummel et al., Challenges for the Wendelstein 7-X Magnet Systems during the next Operation Phase, presented at $27^{\text {th }}$ IEEE SOFE 2017, to be published in Transaction on Plasma Science. 